\title{
On the Comparison of Arctic, Antarctic and Oceanic Atmospheric Electric Measurements
}

\author{
Elmer L. SCHUMAN \\ Atmospheric Physics and Chemistry Laboratory ESSA Research Laboratories \\ Environmental Science Services Administration Boulder, Colorado
}

(Received December 14, 1968)

\begin{abstract}
Data on the diurnal variation of the air-earth current and electric field obtained on the Carnegie ship cruises in 1928-29; at Thule, Greenland in 1958-59; and at the AmundsenScott Station at the South Pole in 1964 are compared from a numerical point of view. The normalized results are found not to agree. The analysis of the degree of significance of the disagreements reveals that different methods of normalizing yield drastically different interpretations; expressing the data as a percentage of the yearly mean renders the differences in the results insignificant at the $75 \%$ level while, if a daily mean is employed, those differences are found to be significant at the $90 \%$ level. The effects of various normalizing procedures on the dispersion of the data are also discussed. How unawareness of the errors arising even in simple averaging of data on atmospheric electric parameters leads to meaningless and often contradictory results is illustrated.
\end{abstract}

\section{Introduction}

In a paper given by Kasemir (1967) at the 1967 IUGG meeting in Lucerne, Switzerland, measurements from 1958 in Thule, Greenland, from 1964 at Amundsen-Scott Station at the South Pole, and from the Carnegie ship cruises are compared as to the universal time variation in the air-earth current and field. The normalized arctic curves were found to be very similar in shape, but with much less amplitude than the oceanic normalizec curves. It is also stated in that paper that this difference cannot be accounted for by measuring error or statistical dispersion of the data and hence must be accepted as real. In the present paper, this difference is examined from a numerical point of view.

Data normalizing procedures are often employed. This is because it is sometimes desirable to compare different parameters (such as field and current) or to compare data of uncertain absolute value obtained by different stations and instruments. One common normalizing procedure consists of expressing the data as a percentage of some mean value. However, whenever mean values are used to represent a group of data, dispersion of the data about the mean value must be considered in the interpretation of the results. The measures of dispersion used in this paper are variance and confidence intervals for means.

\section{Analysis of the Data}

It is first necessary to develop some terminology. There are three sets of data involved: 
(1) hourly means of the electric field from the Carnegie ship cruises in 1928-29 (Torreson et al., 1946), (2) hourly means of the electric field from the Amundsen-Scott Station at the South Pole in 1964 (Kasemir, 1967) and (3) hourly means of the air-earth current from Thule, Greenland in 1958-59 (Kasemir, 1967). Each of these sets of data can best be represented by a matrix $\left(X_{i j}\right)$ where $j=1, \ldots, 24$ symbolizes the UT hour and $i=1, \ldots, N, N$ being the number of complete 24 hour days recorded. Thus $X_{i j}$ is the field (or current) recorded as the mean for the $j$-th UT hour of the $i$-th recorded day.

Consider first a comparison of the Carnegie $(N=179)$ and South Pole $(N=105)$ data. As stated above, some method of normalizing is needed to obtain comparable average hourly values $Y_{j}, j=1, \ldots, 24$. The first method considered computes $Y_{j}$ as:

$$
Y_{j}=\left[100 \cdot \frac{1}{N} \sum_{i=1}^{N} X_{i j}\right] /\left[\frac{1}{24} \sum_{j=1}^{24}\left(\frac{1}{N} \sum_{i=1}^{N} X_{i j}\right)\right] .
$$

This is normalization with respect to a total or "yearly" mean. The results of this calculation (performed by digital computer) are plotted as two curves in Fig. 1 where the difference between them is apparent.

For an analysis of the significance of this difference, a confidence interval for each normalized mean $Y_{j}$ is determined by computing the confidence limits

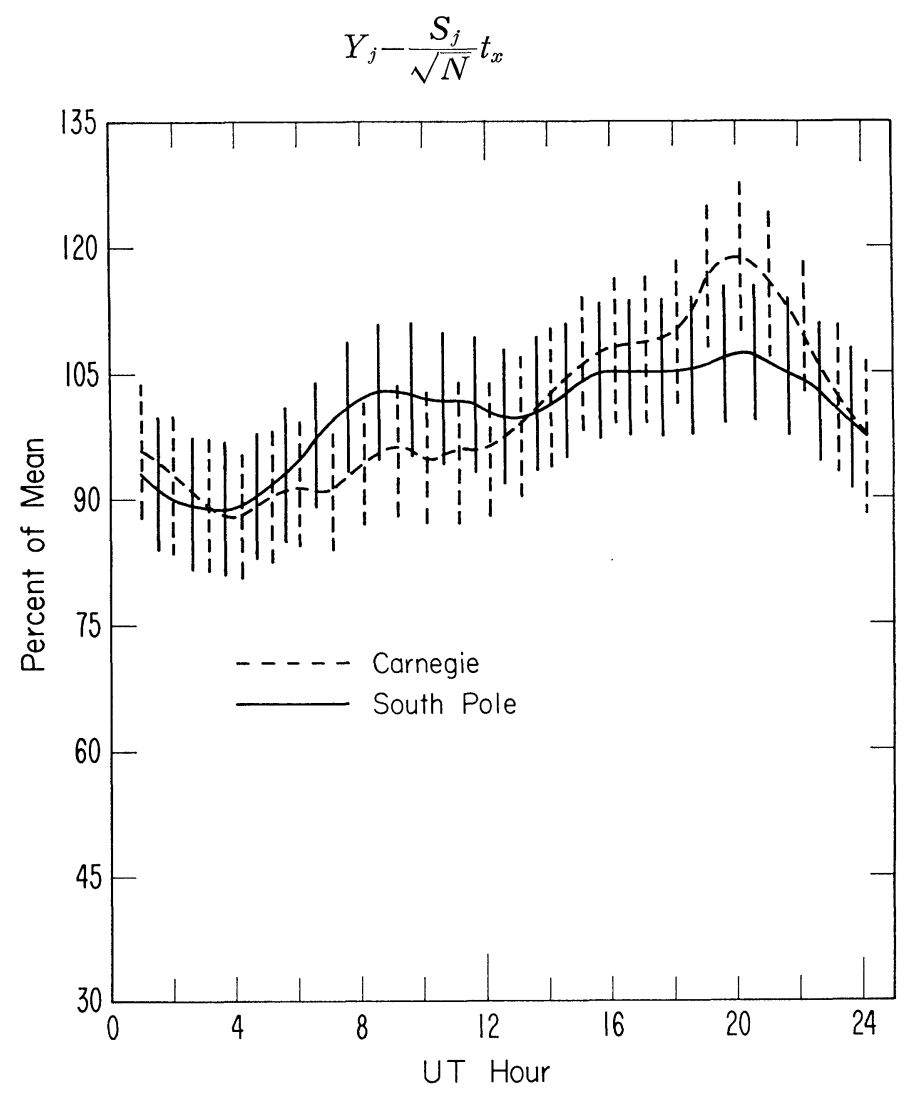

Fig. 1 Carnegie and South Pole data with $75 \%$ confidence intervals when total mean is used. 
and

$$
Y_{j}+\frac{S_{j}}{\sqrt{\bar{N}}} t_{x}
$$

where $S_{j}{ }^{2}$ is the variance given by

$$
S_{j}^{2}=\frac{1}{N} \sum_{i=1}^{N}\left(X_{i j}-\frac{1}{N} \sum_{i=1}^{N} X_{i j}\right) .
$$

and $t_{x}$ is the critical value from the $t$ distribution for $N$ observations, $N$-1 degrees of freedom, at the $x$-th confidence level. The 75\% confidence intervals are shown in Fig. 1 as vertical segments. No significant difference between the two curves is apparent.

The Greenland air-earth current data, although not included in the above analysis exhibits the same behavior.

Consider now a second normalizing procedure, given by the computation

$$
Y_{j}=\frac{1}{N} \sum_{i=1}^{n}\left(100 \cdot X_{i j} / \frac{1}{24} \sum_{j=1}^{24} X_{i j}\right)
$$

With this method, the matrix $\left(X_{i j}\right)$ is transformed before averaging so that each entry becomes normalized with respect to a daily mean. The results of the computation are shown

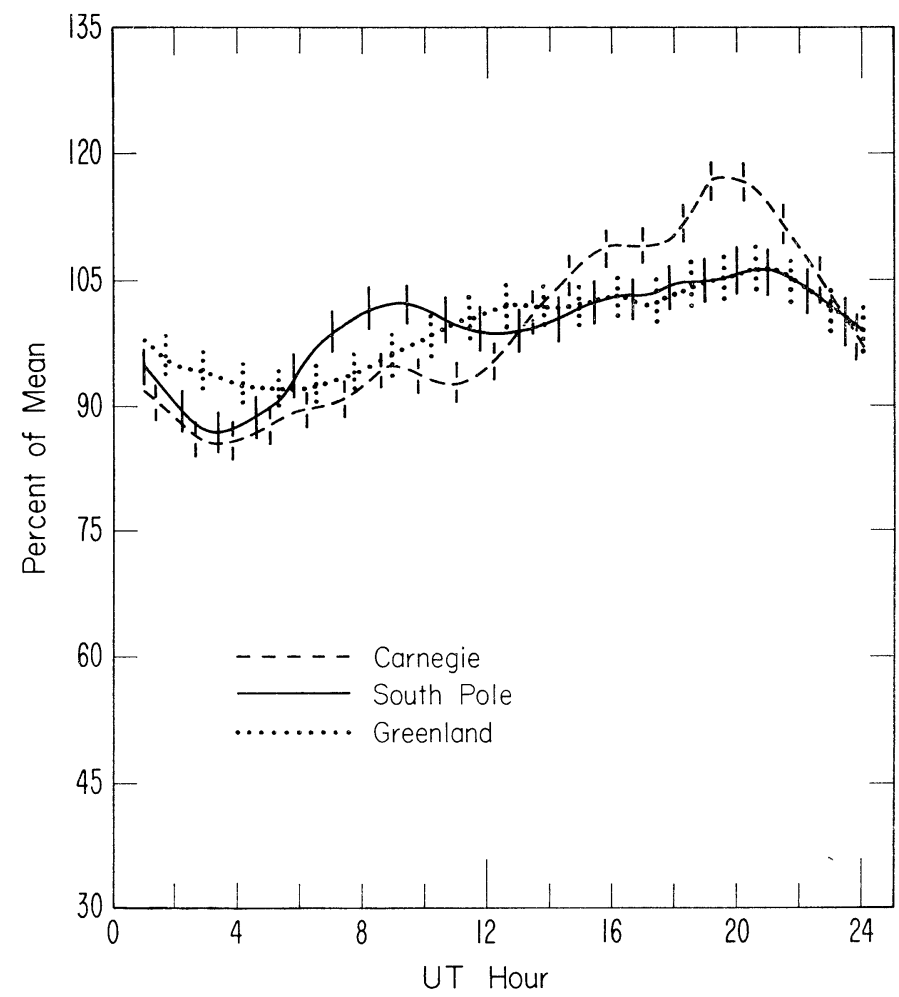

Fig. 2 Carnegie, South Pole, and Greenland data with $90 \%$ confidence intervals when daily mean is used.

Fig. 4 Carnegie, South Pole, and Greenland data with $90 \%$ confidence intervals when continuous daily mean is used. 
in Fig. 2 with $90 \%$ confidence intervals which are, of course, even more stringent than the $75 \%$ intervals used above. The differences between the curves can now be interpreted as significant, in contrast to the results in Fig. 1.

The striking difference between the results of these two normalizing procedures has a physical basis. Results on the diurnal variation, and not on some annual variation, are the main interest. The first normalizing procedure (with respect to a total mean) ignores this fact by not eliminating the day to day annual variations which are irrelevant to the diurnal variations. The second normalizing procedure is specifically designed to eliminate the day to day variations. This explains the smaller variances obtained by the latter method. That there is a large difference between the results of the two methods also has a physical interpretation. It is that a large number of days of data are necessary to obtain meaningful results on the diurnal variation.

It is also important to examine the varian'ces that result from this type of normalization. The variances of $Y_{j}$ for the South Pole data are plotted as an example in Fig. 3. The important feature is that the graph cannot be approximated by a horizontal line. It indicates that the dispersion about the mean is smallest around hour 12 and largest, by as much as

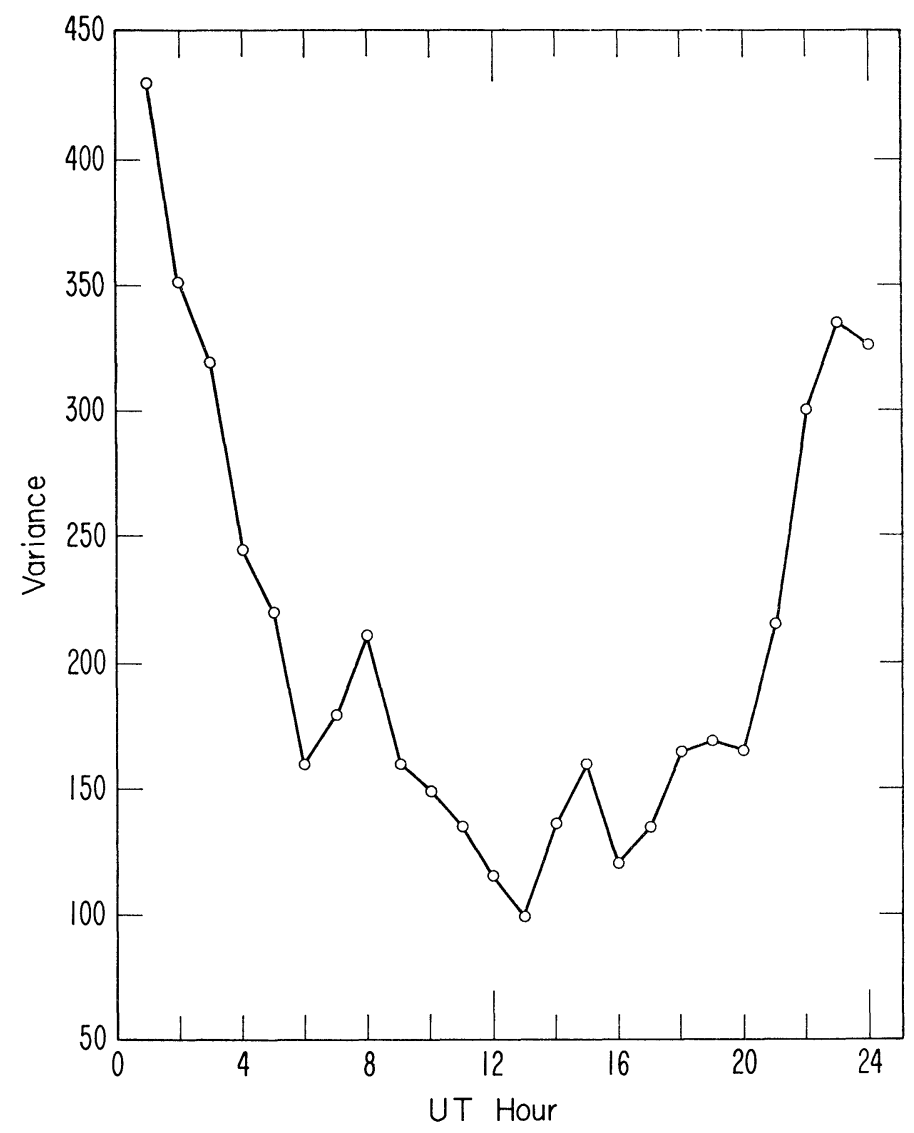

Fig. 3 Variances of the $Y_{j}$ for the South Pole data when daily mean is used. 
a factor of 4, at the ends of the UT day. This has no reasonable physical explanation and is a result of the normalizing process. This normalizing procedure, by employing a daily mean, has created an artificial discontinuity at each point where two consecutive days join. In more detail, within a given day, the diurnal variation is with respect to the mean for that day. The mean for the following day is most probably different, creating a jump at the juncture of the two days. This explains the observed differences in the variances, that is, they are unrealistically high at the ends of the day and low at the center where the values approximate the daily mean.

This situation can be remedied by taking into consideration the day to day trend of the daily means : Let $X_{i j}$ and $X_{i+1, j}$ with $j=1, \ldots 24$ be the data for two consecutive days. The discontinuity occurs between the normalized values of $X_{i, 24}$ and $X_{i+1,1}$. Let $X_{i j} i=n, n+1, \ldots$, $n+m, m>2, j=1, \ldots, 24$ be the data for a sequence of at least three consecutive days. Define

$$
P_{i}=\frac{1}{2}\left(\frac{1}{24} \sum_{j=1}^{24} X_{i j}+\frac{1}{24} \sum_{j=1}^{24} X_{i+1, j}\right)
$$

for $n \leqslant i \leqslant n+m-1$,

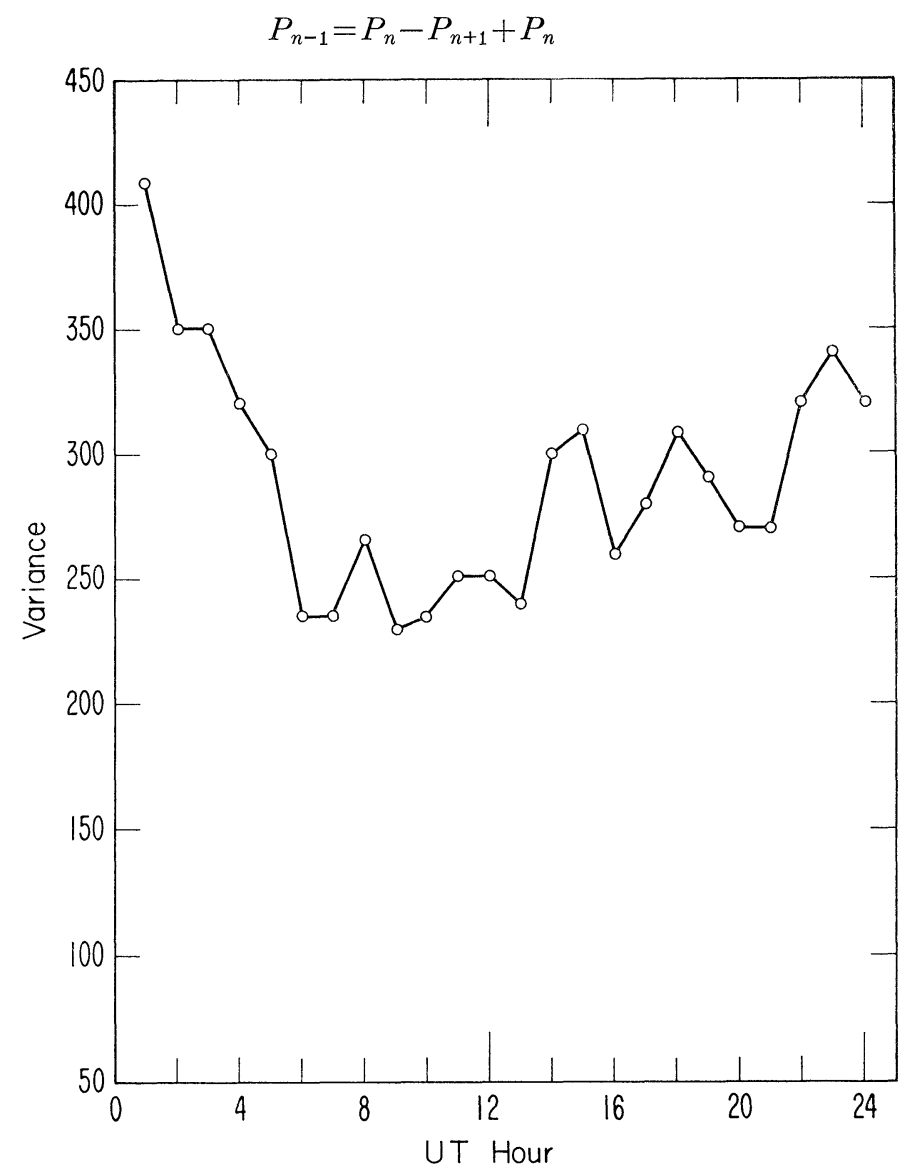

Fig. 5 Variances of the $Y_{j}$ for the South Pole data when continuous daily mean is used. 
and

let

$$
P_{n+m}=P_{n+m-1}-\left(P_{n+m-2}-P_{n+m-1}\right)
$$

$$
M_{i j}=P_{i-1}-\frac{j}{24}\left(P_{i-1}-P_{i}\right)
$$

and transform the matrix $\left(X_{i j}\right)$ into the matrix $\left(X_{i j}^{\prime}\right)$ given by

$$
X^{\prime}{ }_{i j}=100 \cdot X_{i j} / M_{i j}
$$

The transformed matrix $\left(X_{i j}^{\prime}\right)$ represents the diurnal variation with respect to a continuous line determined by the day to day trend of the daily means. For illustration, a portion of this trend line for the South Pole data is given in Fig. 6. The values of $Y_{j}$ are now given by

$$
Y_{j}=\frac{1}{N} \sum_{i=1}^{N} X^{\prime}{ }_{i j}
$$

The results of these calculations for the South Pole, Greenland and Carnegie data are shown in Fig. 4 and are almost identical with the results in Fig. 2. However, a graph of the resulting variances (see Fig. 5) shows that, in contrast to Fig. 3, they provide a more realistic representation for the dispersion of the data.

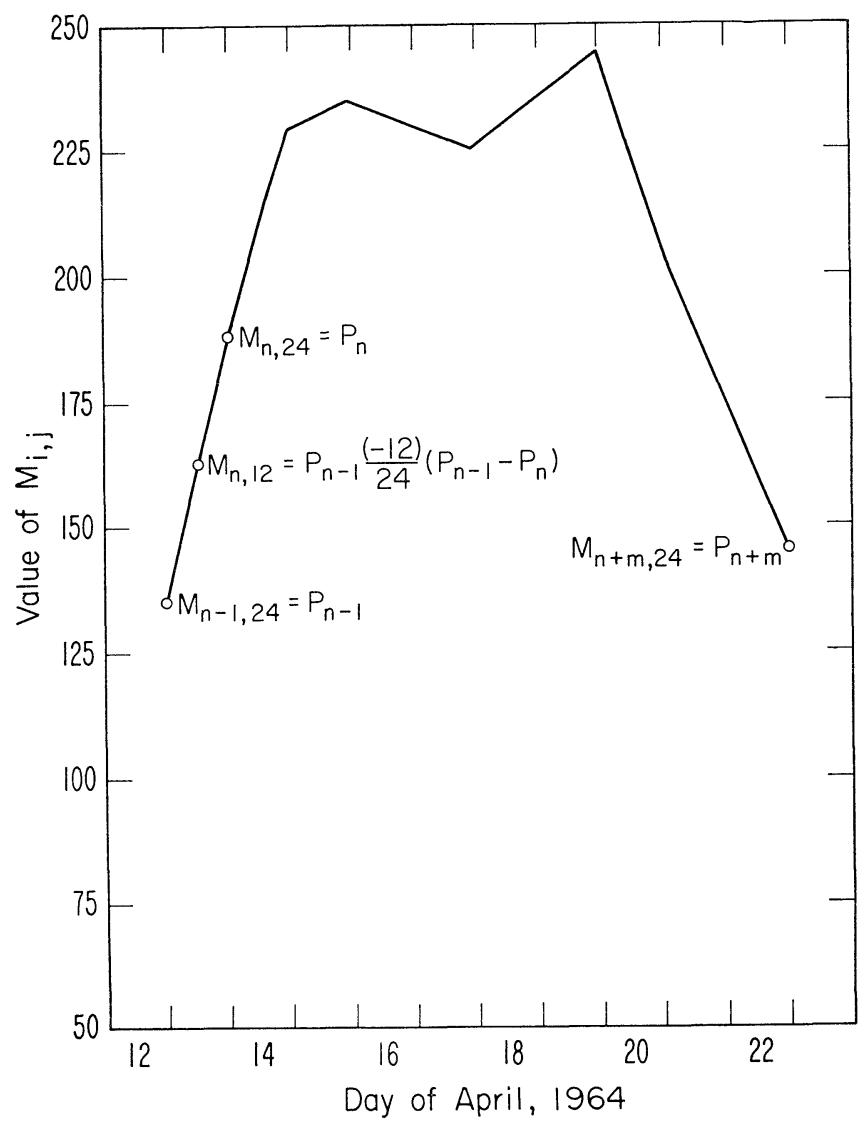

Fig. 6 The non-horizontal nature of the trend in the daily means. 


\section{Conclusion}

In reviewing the above discussion, the reader must be aware of two points. First the arctic and antarctic data differ in certain aspects from the "standard" Carnegie oceanic data. Since this data is at least as reliable as the Carnegie Institute's, this difference casts doubt either on the well established and little questioned Carnegie data or on the concept of a uniform world wide effect of thunderstorm activity on the atmospheric electric parameters.

The second and perhaps more important point is the ease with which atmospheric electric research may lead to meaningless or false results whenever long term data are evaluated. In particular, as is illustrated in this paper, it is indeed meaningless to compare two curves or other data summaries which have been obtained by some type of normalizing or averaging. Examination of the actual Carnegie data revealed that, by simply choosing the proper averaging or normalizing procedure, one may draw any conclusion from comparing measurements with the Carnegie oceanic curves. For example, the three data sets discussed here, when normalized to the total mean show no significant differences even at the small stringency of a $75 \%$ level. However, when a daily mean is used, the differences become significant not only at the $75 \%$ level but even at $90 \%$. The essential point is that whenever data is represented by some type of average or mean, the dispersion of the data determines any interpretation of the results and, if not properly considered, may lead to meaningless conclusions. This is especially true when different sets of data are compared.

With regard to the dispersion of the data, it is illustrated how even such simple procedures as breaking continuous data into daily segments leads to erroneous estimates of dispersion. This of course is reflected in any attempt to determine the significance of results or conclusions.

In summary, it is not the intention of the author to exhibit the complexity of statistics, but to warn about the errors arising even from simple averaging procedures. The results of these errors are, in addition, often irreversible, since it is customary to retain or refer not to original data but only to averaged summaries. This results in the loss of the information needed for estimates of the significance of results.

\section{Acknowledgements}

The author wishes to express his thanks to Dr. H.W. Kasemir for providing the arctic and antarctic data used herein and for his guidance in the presentation of the results.

\section{References}

Kasemir, H.W., Atmospheric Electric Measurement in the Arctic and Antarctic; (Abstract) Int. Assoc. Met. and Atmos. Phys., Report on Proceedings of the XIV General Assembly, Lucerne, Sept. 1967; Publ. IAMAP No. 14, p. 219.

Torreson, O.W., W.D. Parkinson, O.H. Gish, and G.R, Wait; Scientific results of Cruise VII of the Carnegie, ocean atmosphericelectric results ; Carnegie Institution of Washington Publication No. 568, p. $113,1946$. 